This is an electronic reprint of the original article. This reprint may differ from the original in pagination and typographic detail.

Author(s): Kiili, Carita

Title: $\quad$ Argument graph as a tool for promoting collaborative online reading

Year: $\quad 2013$

Version:

Please cite the original version:

Kiili, C. (2013). Argument graph as a tool for promoting collaborative online reading. Journal of Computer Assisted Learning, 29(3), 248-259. https://doi.org/10.1111/j.1365-2729.2012.00492.x

All material supplied via JYX is protected by copyright and other intellectual property rights, and duplication or sale of all or part of any of the repository collections is not permitted, except that material may be duplicated by you for your research use or educational purposes in electronic or print form. You must obtain permission for any other use. Electronic or print copies may not be offered, whether for sale or otherwise to anyone who is not an authorised user. 


\title{
Argument graph as a tool for promoting collaborative online reading
}

\author{
Journal of Computer Assisted Learning
}

Corresponding author:

Carita Kiili

Department of Education, University of Jyvaskyla, Finland

P.O.Box 35, FI- 40014 University of Jyvaskyla, Finland

carita.kiili@jyu.fi 


\title{
Argument graph as a tool for promoting collaborative online reading
}

\begin{abstract}
This study explored how the construction of an argument graph promotes students' collaborative online reading compared to note-taking. Upper secondary school students ( $\mathrm{n}=$ 76) worked in pairs. The pairs were asked to search for and read source material on the Web for a joint essay and either construct an argument graph or take notes during online reading. The data consist of transcripted protocols of student pairs' discussions and joint essays. The study indicated that argument graphs may be useful tools when teachers want students to pay attention to the argumentative content of online sources and to consider relations between arguments. Additionally, with argument graphs teachers can support students' post-reading activities, such as source-based argumentative writing.
\end{abstract}

Keywords: online reading, reading strategies, argument graph, source-based writing

Nowadays, students are often referred to as digital natives (Prensky, 2001) or the net generation (Jones \& Czerniewicz, 2010). However, recent research (Kiili, Laurinen, \& Marttunen, 2008; Walraven, Brand-Gruwel, \& Boshuizen, 2009) has indicated that not all students are that skilled at solving information problems on the Internet. The materials that students encounter on the Internet are diverse in their quality, structure, and argumentation. Therefore, to solve information problems adequately necessitates the ability to identify arguments, evaluate them, and understand how different points of views contradict or corroborate each other. In addition, future working life will increasingly require both online reading and engagement in collaborative literacy practices (Rouet, 2006; Smith, Mikulecky, Kibby, Dreher, \& Dole, 2000). In order to prepare high school students for the world they are entering after school, these literacy skills need to be practiced in schools in authentic online environments (Leu, O’Byrne, Zawilinski, McVerry, \& Everett-Cacopardo, 2009). At the same time, many teachers share a concern that when online sources are allowed in their classrooms students will tend to rely on a superficial copy-paste strategy rather than utilize 


\section{ARGUMENT GRAPH PROMOTING ONLINE READING}

different sources to construct a synthesis on the phenomenon they are exploring. There is a need for collaborative teaching methods that offer opportunities for students to explore in depth and synthesize information when reading multiple online sources. This study investigates whether the construction of an argument graph, as compared to note-taking, promotes students' collaborative online reading, and in particular, their reading to synthesize information.

\section{Online reading comprehension theory}

The online reading comprehension theory defines online reading as a web-based inquiry process (Leu, Kinzer, Coiro, \& Cammack, 2004) which displays notable differences to offline reading comprehension (Coiro \& Dobler, 2007). Effective use of the Internet requires additional reading practices, skills and strategies that readers need to apply flexibly in parallel with traditional ones (Coiro \& Dobler, 2007). At least five processing practices occur during online reading comprehension (Leu et al., 2004): 1) reading to identify important questions, 2) reading to locate information, 3) reading to evaluate information critically, 4) reading to synthesize information, and 5) reading and writing to communicate information. This study explores online reading practices with the focus on reading to synthesize information for the purpose of writing an essay.

\section{Reading to synthesize information during online reading}

Synthesizing can be defined as the creation of an overall meaning by organizing pieces of information and by combining new ideas with earlier interpretations and one's previous knowledge (Keene \& Zimmerman, 2007, p. 229). To be able to create such an overall meaning from one or multiple texts, skilful readers blend claims, arguments, and resources together (Bulger, 2006) by using various reading comprehension strategies, such as 


\section{ARGUMENT GRAPH PROMOTING ONLINE READING}

determining important ideas and combining them, inferencing, and activating one's prior knowledge (Pressley \& Afflerbach, 1995; Eagleton \& Dobler, 2007, p. 204).

Furthermore, while synthesizing usually occurs during reading, the process may also continue after reading (Magliano, Millis, Ozuru, \& McNamara, 2007). This is the case, for example, when students compose an essay on the basis of the source materials they have read. Composing an essay on the basis of multiple sources requires discourse synthesis. By the term discourse synthesis, Spivey and King (1989) refer to a highly constructive process of selecting, organizing, and connecting content from multiple sources when composing a new text. Correspondingly, Segev-Miller (2004) argues that discourse synthesis tasks are cognitively more demanding than summary tasks. When producing a summary from a single text, students can rely on the structure of the original text whereas producing a synthesis from multiple sources requires that they create their own text structure.

Synthesizing is a challenging task for most readers (Rouet, 2006; Mateos \& Sole, 2009) and the Internet introduces new complexities into the synthesizing process (Coiro, 2005). Since solving information problems usually requires that students synthesize information from multiple Web sources they need flexibility in shifting between multiple modes of information (Coiro, 2003; Rouet, 2006) and between different text structures and text genres (Eagleton \& Dobler, 2007). Further, students have to consider how different texts inform or contradict one another (Castek \& Coiro, 2010). The Internet also places additional demands on readers because the argumentative structures of texts on the Web are flexible (Carter, 2003). Identification and analysing arguments has shown to be difficult for students (Larson, Britt, \& Larson, 2004; Marttunen, Laurinen, Litosseliti, \& Lund, 2005) even when they read traditional, linear texts; flexible argument structures of Internet sources makes this even harder. 


\section{ARGUMENT GRAPH PROMOTING ONLINE READING}

In spite of the challenges that Internet sets readers, they can also benefit from exploring multiple sources on the Web. Reading across multiple sources may foster deeper understanding of the phenomenon than reading information from a single source (e.g. Wiley \& Voss, 1999) as it requires that students make an effort to establish and explain connections within and across texts (Wolfe \& Goldman, 2005). Some studies (Wiley \& Voss, 1999; Le Bigot \& Rouet, 2007) indicate that especially argumentative essay tasks facilitate deeper and more integrated text understanding than summary tasks. However, argument tasks are not necessarily optimal for all readers. Students with sophisticated epistemological beliefs may profit more from an argumentative task assignment than students with more naive beliefs (Bråten \& Strømsø, 2010). Most of the studies on reading multiple sources have used printed texts (Wolfe \& Goldman, 2005; Bråten \& Strøms ø, 2010) or the students have worked online with restricted, pre-selected texts (Le Bigot \& Rouet, 2007; Wiley, Goldman, Graesser, Sanchez, Ash, \& Hemmerich, 2009). There is a paucity of research on synthesizing information when students read on the open Internet and on how to support students' synthesizing processes when they read online. This article explores whether the construction of an argument graph helps upper secondary school students to synthesize information when they read online in pairs in order to compose a joint essay.

\section{Argument graph for promoting reading to synthesize}

Theory of representational guidance (Suthers, 2003) frames representational tools as mediators of collaborative learning interactions. Representational tools provide readers with means to represent emerging knowledge and make it visible. The representational tool used in this study was an argument graph (Corbel, Girardot, \& Jaillon, 2002) by means of which students were able to present arguments for and against a specific topic as well as to depict the relations between these arguments graphically. It has been found that argument graphs 


\section{ARGUMENT GRAPH PROMOTING ONLINE READING}

help students to make their thinking visible, to foster consideration of the relations between arguments (Suthers, 2001), and to support productive interaction in collaborative learning situations (Suthers \& Hundhausen, 2003). Furthermore, an argument graph might help students to monitor their progress in the task (Cox, 1999), for example, whether arguments for and against are expressed in a balanced way (Van Drie, Van Boxtel, Jaspers, \& Kanselaar, 2005).

When the Internet is used as an information source, some students just copy and paste material from the Web into their final products. Premier and Ploog (2007) found that students who created a text structure of their own scored significantly higher in the post-test of learning than students who mainly copy-pasted material in their text. The use of an argument graph during successive phases of reading activities might help students to move beyond the copy-paste strategy. An argument graph might help them to re-organize pieces of information (Cox, 1999) and use their own words in connecting the pieces of information together. In the present study, students' collaborative online reading that aims at argumentative source-based writing was supported by an argument graph tool. Potential beneficence of an argument graph was studied by comparing the students who constructed an argument graph with the students who took notes by addressing the following research questions:

1) How did the student pairs in the argument graph and note-taking groups a) engage in online reading practices and $b$ ) what kinds of collaborative reading strategies did they share when exploring a controversial issue?

2) How did the student pairs in the argument graph and note-taking groups synthesize ideas in their essays?

a) What kinds of content did the student pairs include in their joint essays?

b) How did the student pairs synthesize arguments for and against the issue in their essays? 


\section{ARGUMENT GRAPH PROMOTING ONLINE READING}

\section{Methods}

\section{Participants}

Seventy-six upper secondary school students (aged from 16 to 18 years; 47 females, 29 males) volunteered to participate in the present study. The joint essay they wrote compensated for an individual essay that the other students, who did not participate in the study, composed at home.

\section{Task}

The students task was to write an essay on the issue Should Internet censorship be tightened? in pairs by utilizing the Internet as an information source. They were asked to consider arguments both for and against censorship of the Internet. This task assignment was chosen for two reasons. First, it is important for students to learn to carefully evaluate arguments that people or communities with different positions propose on the Internet and weight their argumentation in order to take their own position. Second, the students were asked to search for and ponder arguments both for and against Internet censorship in order to avoid confirmation bias. Namely, it has been reported that if students are asked to express their opinion on a controversial issue prior to reading (Schwarz, 2003), or if they are given a specific viewpoint (against, neutral, for) in advance (Cerdán, Marín, \& Vidal-Abarca, 2011), they tend to focus on reading only those sources or parts of them that support their own opinion or the viewpoint assigned to them.

\section{Procedure}

The activities of this study were integrated into the course on Text and Influence (Finnish Language and Literature). The basics of argumentation were first taught in the class. In the experiment the students worked in pairs. They were allowed to choose their partner freely so that they would feel comfortable in sharing their ideas together (see e.g. Dirks, 1999; 


\section{ARGUMENT GRAPH PROMOTING ONLINE READING}

Kreijns, Kirschner, \& Jochems, 2003). As a result of the self-selection process 20 girl-girl pairs, 11 boy-boy pairs, and 7 girl-boy pairs were formed. The pairs representing each of these gender combinations were randomly divided into two conditions: an argument graph and a note-taking condition.

The researcher met each student pair at a time. The students in the argument graph group were trained to use the Web-based argument graph tool (5 to 10 minutes). With the tool, the students were able to write arguments in boxes, to draw links between the boxes, and to label the links as either supportive (+), critical (-), or neutral (?). The use of the argument graph tool was practiced so that the researcher and the students pondered together arguments for and against the increased use of nuclear power and students formed argument boxes, links and their labels with the tool (Figure 1). The researcher also explained to the students the idea of making argument chains.

\section{---INSERT FIGURE 1 HERE----}

The student pairs worked in three phases. First, the pairs in the argument graph group were asked to discuss the topic and construct an argument graph, while the students in the note-taking group were asked to discuss the topic and take notes (10 to 15 minutes). Second, the student pairs were asked to search for and read additional information on the Web for 30 minutes. The argument graph group was asked to modify their graph and the note-taking group was asked to take paper-and-pencil notes. Finally, the students composed a joint essay (45 minutes).

Throughout the task, the students worked face-to-face. The argument graph group worked with two computers. One computer was used for constructing the graph and for utilizing the graph in the writing phase, and the other computer was available for searching 


\section{ARGUMENT GRAPH PROMOTING ONLINE READING}

for information on the Web and for writing the essay. The note-taking group worked on a single computer. In order to prevent direct copying, the students were not allowed to use a word processor during the information searching phase.

\section{Data}

\section{Interaction protocols}

Interaction protocols (see Miyake, 1986) were analyzed in order to gain access to the online reading practices and collaborative reading strategies that took place during the discussions within each student dyad. A software program was used to capture as video files the discussions and all of the students' web-based activities on the computer screen. Thus, the interaction protocols included information about the Web pages the students visited and the search terms they used in their search queries, as well as their transcribed discussions. During the task, the students visited different kinds of web pages that represented various views on the topic. The students used, for example, news pages, Wikipedia, discussion boards and blogs, interviews, and pages posted by Web communities.

\section{Students' essays}

Following the collaborative reading of online sources and making notes or argument graphs, the students wrote a joint essay $(n=38)$. The essays in the argument graph group comprised on average 273 words $(S D=76)$ and in the note-taking group 271 words $(S D=$ $88)^{1}$

\section{Data analysis of interaction protocols}

\section{Analysis of episodes used to identify online reading practices}

The analysis began with defining and categorizing the online reading episodes. An episode $(n=1043)$ was defined as a thematic entity consisting of successive activities and

\footnotetext{
${ }^{1}$ Since the Finnish language has a highly productive compounding system, a rich derivational system and agglutinative morphology (Aro, 2004) the length of the essays in English would have been more than 400 words.
} 


\section{ARGUMENT GRAPH PROMOTING ONLINE READING}

verbal interactions that served one of the following reading practices: 1) locating information;

2) evaluating information; 3) synthesizing information; 4) monitoring and regulating activities (one's own, other's, or joint activities); and 5) off-task discussions. Analysis of episodes was done taking into account both verbal and screen interaction. The categorization was done according to the primary activity. The length (in seconds) of each episode was measured to determine the total amount of time the student pairs spent on it.

In locating information episodes $(n=312)$ students considered their search strategy, formulated a search query, or chose links from the search results. In evaluating information episodes $(n=154)$ students had to decide whether a certain Web page was worth opening or not. If they opened the page they had to evaluate whether it was reasonable to read it further. The students used credibility or relevance of information as the evaluation criteria. Synthesizing episodes $(n=384)$ consisted of making sense of acquired information by deciding it to be important, making connections between the text and relevant prior knowledge, and/or extending and exploring ideas presented in the text. Monitoring and regulating episodes $(n=180)$ included interactions where the students planned, monitored, regulated, or evaluated either their own, their partner's, or their joint activities. Off-task episodes $(n=13)$ were unrelated to accomplishing the task.

\section{Analysis of utterances to identify collaborative reading strategies}

The analysis continued by defining and categorizing utterances $(n=1891)$ in order to identify the collaborative reading strategies that took place during the synthesizing episodes. The categories of collaborative reading strategies, with examples, are presented in Table 1. The coding reliability of collaborative reading strategies has earlier been found to be $84.5 \%$ between two independent coders (see Kiili, Laurinen, Marttunen, \& Leu, in press). 


\section{Data analysis of the essays}

The essays were analyzed by exploring their content and the arguments presented for and against Internet censorship.

\section{Analysis of the content}

In order to describe the content of the students' essays, each sentence of the essay was classified. Five distinct categories emerged from data: 1) descriptive content, 2) argumentative content, 3) problem solutions, 4) problems, and 5) stance. Table 2 presents examples of the categories. The words included in each category were counted to determine the proportion of each content type in the essay. Two persons coded 6 of the 38 essays (16\%) and achieved $96 \%$ agreement.

\section{---INSERT TABLE 2 HERE----}

\section{Analysis of arguments}

In order to find out how the student pairs presented arguments for and against the issue, the argumentative content of the essays were explored more carefully. All the arguments for and counter-arguments against Internet censorship were identified (see example 1). At first, two persons identified and coded the arguments of 6 essays (16\% of all essays); the level of agreement was $80 \%$. Disagreements were resolved by discussion, after which one person coded the rest of the essays.

\section{Example 1}

Censorship would prevent children from accidently accessing harmful sites [argument for]. On the other hand, the responsibility for children's use of the Internet rests with the children's parents [counter-argument]. However, parents may not always have time [argument for] or they have no interest in dealing with the matter [argument for]. Under these circumstances censorship would be a good thing. 


\section{Results}

\section{Online reading practices}

Table 3 shows that both the argument graph and note-taking groups spent most of their working time on reading to synthesize information and on locating information. The argument graph group spent almost $70.5 \%$ and the note-taking group $65.5 \%$ on reading to synthesize. The corresponding proportions of time spent on locating were $16.4 \%$ and $23.0 \%$. It is noteworthy that the student pairs who constructed argument graphs during online reading spent, on average, less time on locating information than the student pairs who took notes $(U$ $=248 ; p<0.05)$. The average number of locating episodes among the student pairs in the argument graph group was 6.95 whereas in the note-taking group it was $9.47(U=262.5 ; p<$ 0.05). The amount of off-task discussion was low in both groups, indicating that the students concentrated on the task.

---INSERT TABLE 3 HERE----

\section{Collaborative reading strategies}

Both the argument graph and the note-taking groups applied collaborative reading strategies on average almost 50 times (Table 4). Within the both groups, there was a wide variation between the different pairs in the number of occasions on which these strategies were applied. This suggests that between some pairs collaboration was much richer than between other pairs. Gathering information was the most common reading strategy in the both groups. The argument graph group $(M=9.16)$ considered considerably more $(U=16.5$; $p<0.001)$ relations between concepts or arguments than the note-taking group $(M=1.26)$. Putting forward, developing or evaluating arguments was also quite a common strategy in the both groups. This indicates that the argumentative task assignment applied in this study was 


\section{ARGUMENT GRAPH PROMOTING ONLINE READING}

probably successful in directing students' collaborative reading towards argumentative discussion as shown in Example 2. The example also shows how the students came to a mutual agreement by taking into account both arguments for and against the issue at hand.

\section{Example 2}

A: Here is something about racism [gathering information]. We could add that it [Internet censorship] should not be tightened because if racism were not on the Web then it would move in greater extent to reality [putting forward, developing or evaluating arguments]. [The students add the idea into the argument graph].

B: But on the other hand, it [Internet censorship] could decrease racism if racist stuff would not be allowed on the Web then racist thoughts would not necessarily spread that much [putting forward, developing or evaluating arguments].

A: Oh, yeah. Lets' do another box. [The students add aforementioned idea into the graph].

B: People would act in the same way on the Web than they would act in real life. I mean that if you have a sort of ideology, for example, you think that all foreign people are stupid [putting forward, developing or evaluating arguments].

A: Yeah. But on the other hand, if you cannot release your aggression anywhere [putting forward, developing or evaluating arguments].

B: That is true.

Taking notes or constructing an argument graph can be called external reading strategies (e.g. Kobayshi, 2009) that help readers to connect ideas from different sources and recall them later. The argument graph group included, on average, $20.5(S D=6.4)$ argument boxes in their graphs and the note-taking group included $19.0(S D=9.3)$ ideas in their notes that they presented in the most cases with bullets. A little more than half (10/19) of the students collected at least part of their notes into the separate columns of pros and cons. One student pair in the note-taking group constructed a concept map. Interestingly, 6 of 19 student pairs in the note-taking group took double notes, so that both of the students were taking the notes of their own. 


\section{ARGUMENT GRAPH PROMOTING ONLINE READING}

\section{Content of the students' essays}

The students' essays were mainly argumentative in content. The essays of the argument graph group contained more $(U=76 ; p<0.01)$ argumentative content $(M=64.05 \%$ of all content) than those of the note-taking group $(M=44.35 \%)$, as shown in Table 5 . In turn, the essays of the note-taking group were more descriptive in content than those of the argument graph group (23.23\% vs.16.12\%; $U=248 ; p<0.05)$. The note-taking group presented more problems (18.98\% vs. $12.30 \%)$ and solutions to problems (12.15\% vs. $7.22 \%)$ related to Internet censorship than the argument graph group. However, these differences were not statistically significant.

\section{---INSERT TABLE 5 HERE----}

\section{Arguments for and against the issue explored in the students' essays}

The argument graph group presented on average more (11.2 vs. 6.4; SDs are 6.4 and 5.3 respectively) arguments for Internet censorship than the note-taking group ( $U=74.50 ; p$ $<0.01)$. The average total number of arguments was also higher in the essays of the argument graph group (18.3 vs. 11.7; SDs are 7.6 and 5.4) than note-taking group $(U=98.50 ; p<0.05)$. The pairs in the argument graph group presented on average considerably more arguments for than against (11.2 vs. 7.1) Internet censorship $(Z=2.86 ; p<0.01)$. On the contrary, the mean number of arguments for and against (6.4 vs. 5.3) Internet censorship was quite similar in the essays of the note-taking group $(Z=0.94 ; p=$ ns. $)$.

\section{Discussion}

This study indicated that construction of an argument graph may promote students' collaborative online reading and source-based writing in three ways. First, the pairs in the argument graph group spent less time on locating information during online reading than the 


\section{ARGUMENT GRAPH PROMOTING ONLINE READING}

pairs in the note-taking group. This means that the students who used the argument graph had more time to concentrate on reading to synthesize information, which best supports developing an understanding of an issue, than the students who took notes. Since in previous studies (e.g. Henry, 2006) locating information has been shown to be a gate-keeping skill for some online readers, further research is needed to explore in what ways an argument graph can support information search.

Second, the argument graph group considered noticeably more relations between concepts or arguments than the note-taking group during the online reading. This indicates that constructing an argument graph provides students with opportunities to make relations between arguments more explicit. Occasionally, the graph caused the student pairs to negotiate whether a new argument box was related to previously added arguments and whether the new argument supported or opposed the previous arguments. It should be noted that the connections that the students created between the argument boxes on their graph without commenting on them aloud are not included in the present analysis. Thus, in addition to verbalized connections the students were able to share connections through visual means provided by the argument graph tool.

Third, the argument graph group included more argumentative content and more arguments (both for the issue and in overall amount) in their essays than the note-taking group. In line with these results, Jansen, Erkens, and Kirschner (2010) found some positive effects of the use of a Graphical Debate tool on students' source-based writing. The essays of the students who used the tool contained more grounds than the essays of the students who used the Textual Debate tool. They also found that the conceptual quality of the essays of the Graphical Debate group was higher. However, no difference was found in the average quality of the grounds between the groups. 


\section{ARGUMENT GRAPH PROMOTING ONLINE READING}

Compared to the note-taking, constructing an argument graph offers students with opportunities to construct a shared representation. Almost a third of the student pairs in the note-taking group took separate notes of their own whereas the students in the graph group shared one representation on the screen. Compared to paper-pencil notes the construction of a representation on the screen is easier to follow by both students. As the argument graph tool automatically keeps the relations between argument boxes when boxes are added or replaced, it is also easier to keep up-to-date compared to paper-pencil notes.

In addition to the advantages found here, it has previously been found that argument graphs help students to consider arguments for and against in a balanced way (Van Drie et al., 2005). However, the present study did not support this result, as the pairs in the argument graph group presented more arguments for than against the issue in question (11.2 vs. 7.1) whereas note-taking group presented arguments in a more balanced way in the essays (6.4 vs. 5.3). A most obvious reason may be that it was easier to find and elaborate arguments for than against Internet censorship as stated by some of the student pairs in both groups.

Although the arguments were not fully that balanced in the essays of the argument graph group, some of the student pairs counted the number of supporting (+) and critical (-) links directly connected with the main claim. Thus, these pairs were able to utilize the argument graph for monitoring their progress in the task. This potential use of an argument graph could be exploited by giving students more precise instructions with scripts (e.g. Weinberger, Fischer, \& Stegmann, 2005) or metacognitive prompts during their collaborative work with the tool.

Overall, all the student pairs wrote an essay in which the text structure did not follow the same organizational structure as any of the original texts. Thus, none of the pairs, either in the argument graph or in the note-taking group, drew upon a systematic copy-paste strategy. There might be several reasons for this. First, the students had to blend arguments for and 


\section{ARGUMENT GRAPH PROMOTING ONLINE READING}

against the issue, and in order to do this most of the students not only used different sources but also engaged in text-based discussions to elaborate their ideas. Second, the argument graph might have helped the students to re-organize information, use their own forms of expression, and go beyond the meanings of the text. Third, the students in the both groups were not allowed to use a word processor during their information search, and hence were unable to quickly copy-paste text fragments from the Internet. When students use the Internet as an information source, the use of the copy-paste strategy may be prevented by using representational guidance, collaborative work, and by selecting topics that direct students to the use of multiple sources. This may help students to create a text structure of their own (Segev-Miller, 2004) and move from a knowledge telling to a knowledge transforming approach in their writing (Bereiter \& Scardamalia, 1987).

One limitation of this study is that the treatment was very short. Despite this limitation, some important effects of the use of the argument graph were found. Since effective appropriation of a computer tool needs time and practice (De Smet, Broekkamp, Brand-Gruwel, \& Kirschner, 2011), extended time for a writing task, greater specificity of instructions, and a repeated use of the graph might have even emphasized the effects found in this study.

Another limitation of this study is the lack of analysis that would have explored the connections between the interaction protocols and the essays. In the future, deeper analysis is needed to understand how student pairs utilize different sources, such as different web pages, their prior knowledge, and their emerging joint knowledge, when synthesizing information in order to compose a joint essay. The analysis could focus on tracking the sources of each sentence in the students' essays. However, an open Internet environment combined with a collaborative reading situation makes tracking of the origin of sentences in essays more complex compared to closed Internet environment and individual reading situation. This 


\section{ARGUMENT GRAPH PROMOTING ONLINE READING}

complexity could be overcome by using an argument graph as an analytical tool. Interaction protocols would enable the access to both the discussions that student pairs engage in when reading a certain Web page and the formulation of argument boxes related to those discussions. When student pairs then compose their joint essay they probably rely on the content depicted in the argument graph and transfer the ideas from the graph into their essays. In other words, an argument graph could work as a mediator between the fragments in students' essays and the segments of the discussion where the idea presented in the essay was originally read and/or developed.

\section{Concluding remarks}

This study indicated that the argument graph helped the students, in particular, to consider relations between arguments and to make these relations explicit. This demonstrates that with the help of an argument graph students are able to explicate their synthesizing processes more effectively. Furthermore, with argument graphs teachers can support students' post-reading activities, such as source-based argumentative writing. Compared to note-taking the argument graph aided the students to include more argumentative content and arguments in their essays. Thus, constructing an argument graph during online reading may support students in identifying arguments in texts, elaborating them jointly, and including arguments systematically in their joint essay. Additional advantages may be achieved with more thorough and explicit instructions and by using the argument graph tool several times during lessons. As many other digital computer tools, the argument graph tool is technically easy to use. Therefore, support for students on the appropriate use of the argument graph should, instead of technical matters, rather focus on higher-order thinking skills, such as understanding the argumentative structure and relations between the arguments in a text. 


\section{ARGUMENT GRAPH PROMOTING ONLINE READING}

\section{References}

Aro, M. (2004). Learning to read: The effect of orthography. Jyväskylä Studies in Education, Psychology and Social research, 237. Jyväskylä, Finland: University of Jyväskylä.

Bereiter, C., \& Scardamalia, M. (1987). The psychology of written composition. Hillsdale, NJ: Lawrence Erlbaum.

Bråten, I., \& Strømsø, H. I. (2010). Effects of task instruction and personal epistemology on the understanding of multiple texts about climate change. Discourse Process, 47(1), 1-31.

Bulger, M. (2006). Beyond search: A preliminary skill set for online literacy. Published online by the Transliteracies Project, University of California, Santa Barbara. Retrieved from http://transliteracies.english.ucsb.edu/research-papers/ translit-bulger-onlinelit.pdf

Carter, L. (2003). Argument in hypertext: Writing strategies and the problem of order in a non-sequential world. Computers and Composition, 20(1), 3-22.

Castek, J., \& Coiro, J. (2010). Measuring online reading comprehension in open networked spaces: Challenges, concerns, and choices. Poster presented at Annual Meeting of American Educational Research Association, Denver, April 30 - May 4, 2010.

Cerdán R., Marín, M.C., \& Vidal-Abarca, E. (2011). Perspective and source effects when high-school students read multiple contradictory documents. Paper presented at the 13th Biennial Conference Earli 2011, Exeter, UK, August 30 - September 3, 2011.

Coiro, J. (2003). Reading comprehension on the Internet: Expanding our understanding of reading comprehension to encompass new literacies. The Reading Teacher, 56(5), 458-464.

Coiro, J. (2005). Making sense of online text. Educational Leadership, 63(2), 30-35.

Coiro, J., \& Dobler, E. (2007). Exploring the online reading comprehension strategies used by sixth-grade skilled readers to search for and locate information on the Internet. Reading Research Quarterly, 42(2), 214-257.

Corbel, A., Girardot, J., \& Jaillon, P. (2002). DREW: a dialogical reasoning web tool. In A. Mendes Villas, J. A. Mesa Gonzalez, \& I. Solo de Zaldivar (Eds.), Educational technology: Vol 1. Proceedings of the international conference on ICT's in education (ICTE 2002) (pp. 516-521). Badajoz, Spain: Junta de Extremadura, Consejeria de education, cienca y technologia.

Cox, R. (1999). Representation construction, externalized cognition and individual differences. Learning and Instruction, 9(4), 343-363.

De Smet, M. J. R., Broekkamp, H., Brand-Gruwel, S., \& Kirschner, P. (2011). Effects of electronic outlining on students' argumentative writing performance. Journal of Computer Assisted Learning, 27(6), 557-574.

Dirks, K. T. (1999). The effects of interpersonal trust on work group performance. The Journal of Applied Psychology, 84(3), 445-455. 
Eagleton, M. B., \& Dobler, E. (2007). Reading the Web: Strategies for Internet inquiry. New York: The Guilford Press.

Henry, L. A. (2006). SEARCHing for an answer: The critical role of new literacies while reading on the Internet. The Reading Teacher, 59(7), 614-627.

Janssen, J., Erkens, G., \& Kirschner, P. A. (2010). Effects of representational guidance during computer-supported collaborative learning. Instructional Science, 38(1), 59-88.

Jones, C., \& Czerniewicz, L. (2010). Describing or debunking? The net generation and digital natives. Journal of Computer Assisted Learning, 26(5), 317-320.

Keene, E. O., \& Zimmerman, S. (2007). Mosaic of thought: The power of comprehension strategy $\left(2^{\text {nd }}\right.$ ed.). Portsmouth, NH: Heinemann.

Kiili, C., Laurinen, L., \& Marttunen, M. (2008). Students evaluating Internet sources: From versatile evaluators to uncritical readers. Journal of Educational Computing Research, 39(1), 75-95.

Kiili, C., Laurinen, L., Marttunen, M., \& Leu, D. J. (in press). Working on understanding during collaborative online reading. Journal of Literacy Research.

Kreijns, K., Kirschner, P. A., \& Jochems, W. (2003). Identifying the pitfalls for social interaction in computer-supported collaborative learning environments: A review of the research. Computers in Human Behavior, 19(3), 335-353.

Kobayashi, K. (2009). Comprehension of relations among controversial texts: Effects of external strategy use. Instructional Science, 37(4), 311-324.

Larson, M., Britt, M. A., \& Larson A. A. (2004). Disfluencies in comprehending argumentative texts. Reading Psychology, 25(3), 205-224.

Le Bigot, L., \& Rouet, J. F. (2007). The impact of presentation format, task assign- ment, and prior knowledge on students' comprehension of multiple online documents. Journal of Literacy Research, 39(4), 445-470.

Leu, D. J., Kinzer, C. K, Coiro, J. L, \& Cammack, D. W. (2004). Toward a theory of new literacies emerging from the Internet and other information and communication technologies. In R. B. Ruddell, \& N. Unrau (Eds.), Theoretical models and process of reading ( $5^{\text {th }}$ ed., pp. 1570-1613). Newark, Del: International Reading Association.

Leu, D. J., O’Byrne, W. I., Zawilinski, L., McVerry, J. G., \& Everett-Cacopardo, H. (2009). Expanding the New Literacies Conversation. Educational Researcher, 38(4), 264-269.

Magliano, J. P., Millis, K., Ozuru, Y., \& McNamara, D. S. (2007). A multidimensional framework to evaluate reading assesment tools. In D. S. McNamara (Ed.) Reading comprehension strategies: Theories, interventions and technologies. (pp. 107-136). New York: Lawrence Erlbaum Associates. 


\section{ARGUMENT GRAPH PROMOTING ONLINE READING}

Marttunen, M., Laurinen, L., Litosseliti, L., \& Lund, K. (2005). Argumentation skills as prerequisites for collaborative learning among Finnish, French and English secondary school students. Educational Research and Evaluation, 11(4), 365-384.

Mateos, M., \& Solé, I. (2009). Synthesising information from various texts: A study of procedures and products at different educational levels. European Journal of Psychology of Education, 24(4), 435-451.

Miyake, N. (1986). Constructive interaction and the iterative process of understanding. Cognitive Science, 10(2), 151-177.

Premier, B., \& Ploog, M. (2007). The influence of text production on learning with the Internet. British Journal of Educational Technology, 38(4), 613-622.

Prensky, M. (2001). Digital natives, digital immigrants part 1. On the Horizon, 9(5), 1-6.

Pressley, M., \& Afflerbach, P. (1995). Verbal protocols of reading: The nature of constructively responsive reading. Hillsdale, N.J.: Lawrence Erlbaum.

Rouet, J. F. (2006). The skills of document use: From text comprehension to Web-based learning. Mahwah, NJ: Erlbaum.

Schwarz, B. (2003). Collective reading of multiple texts in argumentative activities. International Journal of Educational Research, 39(1-2), 133-151.

Segev-Miller, R. (2004). Writing from sources: The effect of explicit instruction on college students' processes and products. L1 - Educational Studies in Language and Literature, 4(1), $5-33$.

Smith, M. C., Mikulecky, L., Kibby, M. W., Dreher, M. J., \& Dole, J. A. (2000). What will be the demands of literacy in the workplace in the next millennium? Reading Research Quarterly, 3(3), 378-383.

Spivey, N. N., \& King, J. R. (1989). Readers as writers composing from sources. Reading Research Quarterly, 24(1), 254-277.

Suthers, D. D. (2001). Towards a systematic study of representational guidance for collaborative learning discourse. Journal of Universal Computer Science, 7(3), 254-277.

Suthers, D. D. (2003). Representational guidance for collaborative inquiry. In J. E. B.

Andriessen, M. Baker, \& D. D. Suthers (Eds.), Arguing to learn. Confronting cognitions in computer-supported collaborative learning environments (pp. 27-46). Dordrecht: Kluwer.

Suthers, D. D., \& Hundhausen, C. D. (2003). An Experimental Study of the Effects of Representational Guidance on Collaborative Learning Processes. The Journal of the Learning Sciences, 12(2), 183-218.

Walraven, A., Brand-Gruwel, S., \& Boshuizen, H. P. A. (2009). How students evaluate information and sources when searching the World Wide Web for information. Computers \& Education, 52(1) 234-246. 


\section{ARGUMENT GRAPH PROMOTING ONLINE READING}

Van Drie, J., Van Boxtel, C., Jaspers, J., \& Kanselaar, G. (2005). Effects of representational guidance on domain specific reasoning in CSCL. Computers in Human Behavior, 21(4), 575602.

Weinberger, A., Fischer, F., \& Stegmann, K. (2005). Computer-Supported Collaborative Learning in higher education: Scripts for argumentative knowledge construction in distributed groups. In T. Koschmann, D. Suthers, T. W. Chan (Eds.), Proceedings of Computer Supported Collaborative Learning 2005: The Next 10 Years! (pp. 717-726). Lawrence Erlbaum Associates, Mahwah, NJ.

Wiley, J., Goldman, S. R., Graesser, A. C., Sanchez, C. A., Ash, I. K., \& Hemmerich, J. A. (2009). Source Evaluation, Comprehension, and Learning in Internet Science Inquiry Tasks. American Educational Research Journal, 46(4), 1060-1106.

Wiley, J., \& Voss, J. F. (1999). Constructing arguments from multiple sources: Tasks that promote understanding and not just memory for text. Journal of Educational Psychology, 91(2), 301-311.

Wolfe, M. B. W., \& Goldman, S. R. (2005). Relations between adolescents' text processing and reasoning. Cognition and Instruction, 23(4), 467-502. 


\section{ARGUMENT GRAPH PROMOTING ONLINE READING}

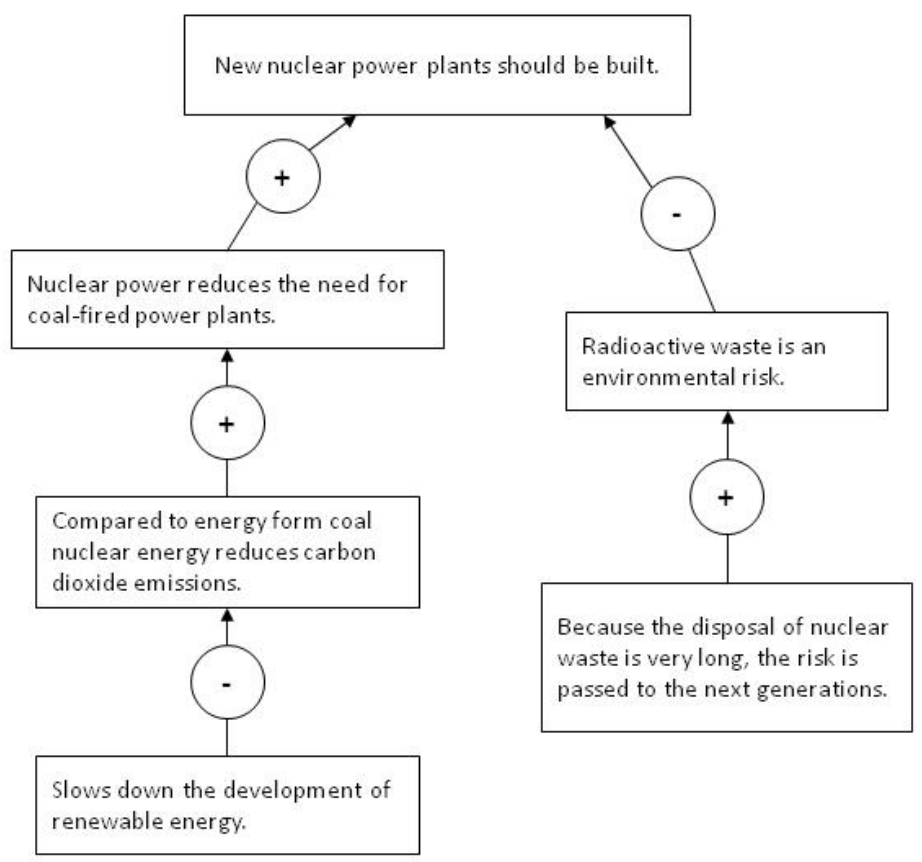

Figure 1. An example of an argument graph on nuclear power 


\section{ARGUMENT GRAPH PROMOTING ONLINE READING}

Table 1. Collaborative reading strategies.

Collaborative reading strategy Example

Gathering information (e.g. facts, arguments)

Considering relations between concepts and arguments

Recapitulating information

Using prior knowledge

Inferencing

Proposing solutions

Asking questions on the topic

Expressing opinion or disagreement

Putting forward, developing, or evaluating arguments
The Ministry of Social Affairs and Health suggested that online poker sites should be censored. They would censor them totally.

It [difficulty to define what should be censored] is slightly related to a 'police state' or to 'freedom of speech is violated'.

Leena: They should be censored more carefully [related the discussion on school shootings]

Mari: So, these sites that young people constantly visit, for example, Galleria or YouTube

Leena: Thus, the web sites that are favored by youth should be censored, if needed.

But in China they censor the Internet a lot.

Right. Censorship has a long tradition.

In my opinion filters should be used. [Filters] should be installed more.

Then, what should be censored and why?

Although I disagree with that [censorship gives too much power to the police].

Well. Let say at least that censoring religious issues is against the freedom of worship [an example of an argument against Internet censorship]. 


\section{ARGUMENT GRAPH PROMOTING ONLINE READING}

Table 2. Content categories of the essays.

\begin{tabular}{ll}
\hline Content category & Example \\
\hline Descriptive content & $\begin{array}{l}\text { The purpose of Internet censorship is to prevent publication of } \\
\text { pictures or texts that are illegal or harmful. }\end{array}$ \\
Argumentative content & $\begin{array}{l}\text { For example, by tightening censorship rapid spread of racist } \\
\text { ideas on the Web could be prevented. }\end{array}$ \\
Problem solutions & $\begin{array}{l}\text { Internet operators should provide services for families with } \\
\text { children that would inhibit children's access to Web sites } \\
\text { prohibited to them. }\end{array}$ \\
Problems & $\begin{array}{l}\text { The Internet is spread so widely that in practice it is almost } \\
\text { impossible to fully control it. }\end{array}$ \\
Stance & $\begin{array}{l}\text { Censorship can be tightened, as long as it is done legally and } \\
\text { with respect for human rights. }\end{array}$ \\
\hline
\end{tabular}




\section{ARGUMENT GRAPH PROMOTING ONLINE READING}

Table 3. Mean numbers of episodes and proportions of time spent on different online reading practices in the argument graph and note-taking groups.

\begin{tabular}{|c|c|c|c|c|c|c|c|c|}
\hline \multirow[b]{3}{*}{ Online reading practice } & \multicolumn{4}{|c|}{ Argument graph group $(n=19)$} & \multicolumn{4}{|c|}{ Note-taking group $(\mathrm{n}=19)$} \\
\hline & \multicolumn{2}{|c|}{$\begin{array}{l}\text { Number of } \\
\text { episodes }\end{array}$} & \multicolumn{2}{|c|}{$\begin{array}{l}\text { Time spent on online } \\
\text { reading practice }\end{array}$} & \multicolumn{2}{|c|}{$\begin{array}{l}\text { Number of } \\
\text { episodes }\end{array}$} & \multicolumn{2}{|c|}{$\begin{array}{l}\text { Time spent on online } \\
\text { reading practice }\end{array}$} \\
\hline & $M$ & $S D$ & $M \%$ & $S D$ & $M$ & $S D$ & $M \%$ & $S D$ \\
\hline Synthesizing information & 9.95 & 2.61 & 70.52 & 8.44 & 10.26 & 2.81 & 65.48 & 13.20 \\
\hline Locating information & $6.95^{1}$ & 2.92 & $16.38^{2}$ & 7.35 & $9.47^{1}$ & 2.99 & $22.97^{2}$ & 10.64 \\
\hline $\begin{array}{l}\text { Monitoring and } \\
\text { regulating activities }\end{array}$ & 4.58 & 2.52 & 7.52 & 4.88 & 4.89 & 3.02 & 6.55 & 5.12 \\
\hline Evaluating information & 3.37 & 3.69 & 3.39 & 4.27 & 4.74 & 2.66 & 4.71 & 3.67 \\
\hline Off-task & 0.47 & 1.07 & 2.20 & 6.39 & 0.21 & 0.54 & 0.29 & 0.79 \\
\hline Total & $25.32^{3}$ & 7.34 & 100.01 & & $29.57^{3}$ & 7.19 & 100.00 & \\
\hline
\end{tabular}

${ }^{1} U=262.5, p<0.05 ;{ }^{2} U=248, p<0.05 ;{ }^{3} U=253, p<0.05$ 


\section{ARGUMENT GRAPH PROMOTING ONLINE READING}

Table 4. Collaborative reading strategies used in the argument graph and note-taking groups.

\begin{tabular}{|c|c|c|c|c|}
\hline \multirow[b]{2}{*}{ Collaborative reading strategy } & \multicolumn{2}{|c|}{$\begin{array}{l}\text { Argument graph } \\
\text { group }(n=19)\end{array}$} & \multicolumn{2}{|c|}{$\begin{array}{l}\text { Note-taking group } \\
\quad(n=19)\end{array}$} \\
\hline & $\mathrm{M}$ & SD & M & SD \\
\hline Gathering information & 14.95 & 6.63 & 18.42 & 9.47 \\
\hline $\begin{array}{l}\text { Considering relations between concepts or } \\
\text { arguments }^{1}\end{array}$ & 9.16 & 5.61 & 1.26 & 1.73 \\
\hline Putting forward, developing or evaluating arguments & 7.79 & 8.99 & 7.95 & 8.00 \\
\hline Inferencing & 5.42 & 4.05 & 5.84 & 3.92 \\
\hline Using prior knowledge & 4.00 & 3.84 & 5.74 & 4.53 \\
\hline Asking question on the topic & 3.95 & 3.47 & 4.53 & 6.21 \\
\hline Recapitulating information & 2.32 & 2.19 & 1.16 & 1.21 \\
\hline Expressing an opinion or disagreement & 1.89 & 2.26 & 2.63 & 2.50 \\
\hline Proposing a solution & 0.37 & 0.76 & 2.16 & 3.80 \\
\hline Total & 49.85 & 21.38 & 49.69 & 30.46 \\
\hline
\end{tabular}

${ }^{1} U=16.5, p<0.001$ 


\section{ARGUMENT GRAPH PROMOTING ONLINE READING}

Table 5. Proportions of types of content of the students' essays.

\begin{tabular}{lrrrrr}
\hline & \multicolumn{2}{c}{ Argument graph group } & & \multicolumn{2}{c}{ Note-taking group } \\
\cline { 2 - 3 } \cline { 5 - 6 } Content type & $M \%$ & $S D$ & & $M \%$ & $S D$ \\
\hline Argumentative $^{1}$ & 64.05 & 23.57 & & 44.35 & 18.88 \\
Descriptive $^{2}$ & 16.12 & 12.72 & & 23.23 & 12.46 \\
Problems & 12.30 & 10.45 & & 18.98 & 14.38 \\
Solutions & 7.22 & 13.25 & & 12.15 & 10.86 \\
Stance & 0.32 & 1.06 & & 1.29 & 3.84 \\
Total & 100.01 & & & 100.00 & \\
\hline
\end{tabular}

${ }^{1} U=76, p<0.01 ;{ }^{2} U=248, p<0.05$ 